\title{
Transatlantic exchange programme between critical care nurses
}

Jan Weststrate, RN, CCRN, MSc, Clinical Researcher, Department of General Surgery, Erasmus MC, University Hospital, Rotterdam, The Netherlands

JTM Weststrate, Erasmus MC, University Hospital Rotterdam, Room Z-638, PO Box 2040, 3000 CA, Rotterdam, The Netherlands. e-mail: weststrate@aziv.azr.nl

\section{BACKGROUND TO PROGRAMME}

Since 1998, the University Hospital Rotterdam has run an exchange programme for critical care nurses with the Strong Memorial Hospital in Rochester, New York, USA. In this programme, a group of nurses from both hospitals, who work in the adult, neonatal and paediatric intensive care units (ICUs), visit each other for one week every year.

The Dutch group visits Strong Memorial Hospital for a week at the beginning of October, while the Americans visit Holland in April as the spring bulbs are just starting to flower. In order to stimulate exchange in the broadest sense, nurses stay not in a hotel but with their colleagues at home. In principle, the Dutch nurses stay with the colleagues who will be staying with them the following April.

\section{APPLICATION TO JOIN PROGRAMME}

Nurses have to submit an application to their nurse manager to become part of the exchange programme. This application is accompanied by an essay in which the critical care nurse explains why this exchange programme is important for themselves, their ICU and their hospital. Each year, one nurse from each unit is allowed to take part in the programme.

\section{EXCHANGE PROGRAMME SCHEDULE}

The exchange programme has now been running for four years. The schedule for the week has been established as follows and is the same in both countries.

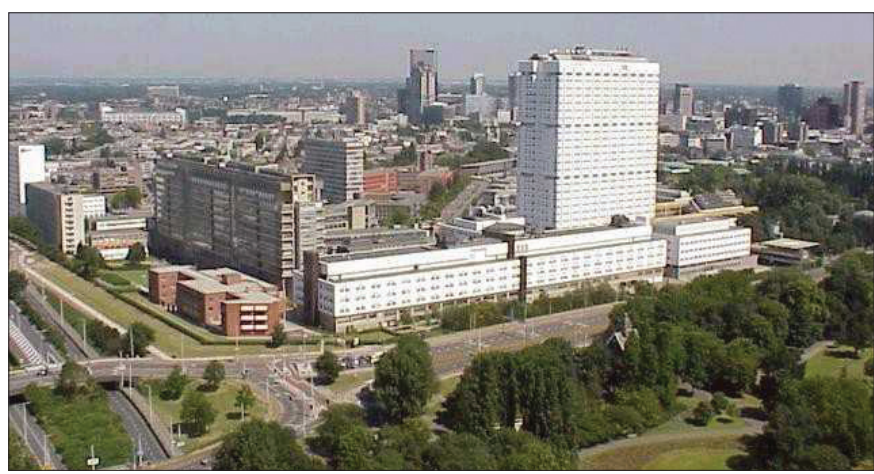

Erasmus MC University Hopsital, Rotterdam, The Netherlands.

\section{Monday}

On Monday, the participants are introduced to the health care system in the country they are visiting. The introduction is given by a several different speakers who discuss different health care issues related to critical care. In the afternoon, the group is given a tour throughout the hospital, which includes visits to the different ICUs. This is often the first encounter of the visiting group with critical care nursing in the country being visited. Numerous questions are always asked. By the evening, the participants are ready to relax after their busy day with a social evening and dinner.

\section{Tuesday}

On their second day, the participants act as a shadow nurse with their host on the visiting ICUs. This gives them a good impression of how the work on the unit is organised and which aspects are different compared with their own unit. The Tuesday evening is spent quietly with the host nurse, as the participants are often exhausted after all the new impressions and information they have received during the day.

\section{Wednesday}

Wednesday is the day set aside for presentations. Each of the visiting nurses gives a slide presentation, using a Power Point program, on a topic that is relevant for them or their own ICU, in relation to critical care nursing. This aspect of the exchange programme is always the one most dreaded by the visiting nurses, particularly for the Dutch nurses who must give their presentation in English. Often, it is the first time the Dutch participants have given a presentation in front of other col-

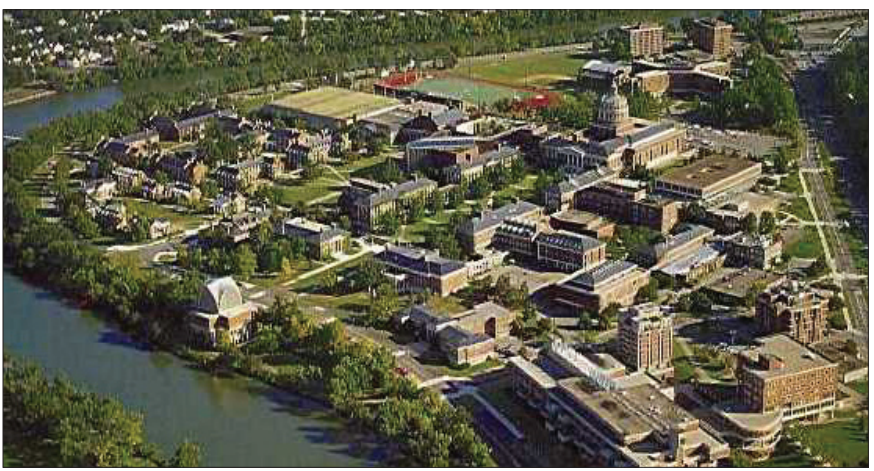

Strong Memorial Hospital and its University at Rochester, NY, USA. 


\section{Working in Iceland ICUs compared with UK ICUs}

Anna Lóa Magnúsdóttir, BSc in Nursing and Diploma in Critical Care Nursing, Intensive Care Unit, Landspítalinn, University Hospital, Fossvogi, Reykjavík, Iceland (-

Arnfrídur Magnúsdóttir, BSc in Nursing and Diploma in Critical Care Nursing, Intensive Care Unit, Landspítalinn, University Hospital, Fossvogi, Reykjavík, Iceland

Anna Lóa Magnúsdóttir, Gjorgaesludeild (Intensive Care Unit), Landspítalinn, University Hospital, Fossvogi 108 Reykjavík, Iceland. e-mail: annaloa@vortex.is

\section{INTRODUCTION}

In the year 2000, as part of our postgraduate programme in critical care nursing, we spent four months in England working in four different intensive care units (ICUs). These were an adult ICU, a coronary care unit, a paediatric ICU and a neonatal ICU.

When working in a new country, we expected things to be done differently to the way we were used to. There are many factors that can influence the way things are done, including education, health care system, culture, and size of the country and population. In this article, we have written about our experiences of the differences in critical care nursing between England and Iceland.

\section{INTENSIVE CARE IN ICELAND}

Iceland is an island in the north Atlantic Ocean. It has a small population of about 300,000 people. In spite of our small population size, we have a high standard of living and a highquality health care system.

\section{Critical care training}

In Iceland, there are two universities which produce graduate nurses. A nursing graduate education takes four years to complete and results in a bachelor's degree. A course in critical care nursing is available, but is not held on a regular basis; it results in a diploma in critical care nursing. It is necessary to go abroad to obtain a masters' degree in critical care nursing. This situation is likely to change in the next few years. However, at present, from a hospital's point of view, it is not necessary in Iceland for a nurse to have a diploma or degree in critical care nursing to work as an ICU nurse.

\section{Provision of critical care}

We worked on the mixed adult/paediatric ICU at the university hospital in Reykjavik; the unit was a mixed medical, surgical, trauma and neurosurgical unit. In the whole of Iceland, there are only three other ICUs, one of which is for neonates. The other two ICUs are mixed adult/paediatric units, one of which is also in the capital city of Reykjavik, while the other is in Akureyri, the biggest town in northern Iceland.

\section{COMPARISON OF INTENSIVE CARE BETWEEN UK AND ICELAND}

The units we visited in the UK were smaller than the ICU in Reykjavik. In the UK, ICUs have between 6 and 8 beds per unit, while our unit in Reykjavik had 11 beds. The units in the UK had more general specialities, such as for vascular surgery, neurological surgery, cardiac and thoracic surgery. In the UK, patients were sent to hospitals according to the type of help needed.

In Iceland, ICUs are more general. However, the two ICUs in Reykjavik do have their own specialities, although both still remain general medical/surgical ICUs looking after patients of all ages.

\section{Nurse:patient ratio and workload}

The patient to nurse ratio was better at the ICUs we visited in the UK than at our ICU in Iceland. The UK ICUs had one nurse per patient, except in the neonatal ICU where the nurses often had more than one neonate to care for. Furthermore, UK ICUs had one sister or senior staff nurse in charge of every shift without responsibility for patient care.

In our unit in Iceland, we usually tried to have a ratio of one nurse per patient, but often, especially on nights and weekends, this was not possible. The nurse in charge of the shift nearly always had a patient to care for as well.

In addition, the post-anaesthesia unit was linked to our ICU. The ICU nurses were responsible for the care of these patients at nights and weekends, which sometimes took time from the ICU patient, and often increased the workload.

\section{Admitting patients to an ICU}

In the UK ICUs, we noticed that they did not admit a new patient to the unit unless enough nurses were available. Patients were sent to other hospitals if no beds or nurses were available.

In Iceland, our unit in Reykjavik had to take every patient who needed care in an ICU, independent of the nurses available, because there are not the resources to be able to send a patient to another ICU. However, if no bed was available and there was no patient who could be discharged, it was sometimes possible to send a patient to the other ICU in Reykjavik, provided of course that they had an available bed. 


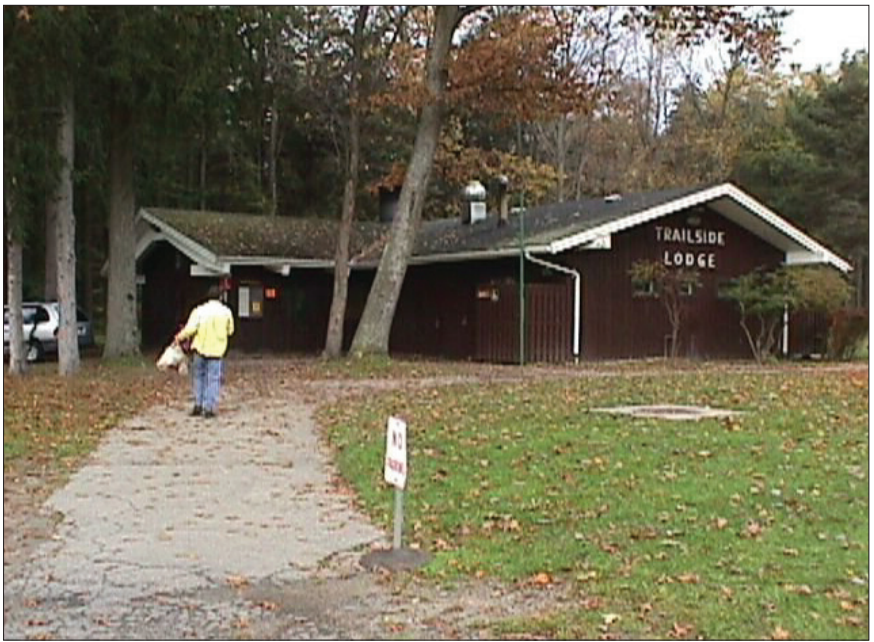

'The research cabin' in Letchworth Park, NY, USA.

leagues, never mind the fact that it also has to be done in English!

After the presentations, everybody is relieved that all has gone well and they are more than ready for some fun. Thus, the rest of the day is filled with shopping or being a tourist.

\section{Thursday}

'Research day' is Thursday. As there is an interval of 5-6 months between the exchange visits, the participants gather data during this period around a subject strongly related to nursing practice. The aim is to compare the gathered data between the two hospitals on the second visit in order to detect differences and commonalities. In the discussion, it is always interesting to hear the different arguments in relation to 'Why?'-type questions. So far, we have gathered data between the two hospitals about varying issues, such as interventions to prevent pressure ulcers and endotracheal suctioning. The research day is always held at a different, special location, away from the hospital, such as a cabin in a national park in the Rochester area or an 1,000-yearold castle in the Netherlands.

\section{Friday}

Friday is the last official day of the exchange programme and is known as the 'cultural day'. Participants and host nurses visit a cultural place and reflect on what they have experienced during the past week from a professional and personal point. In the

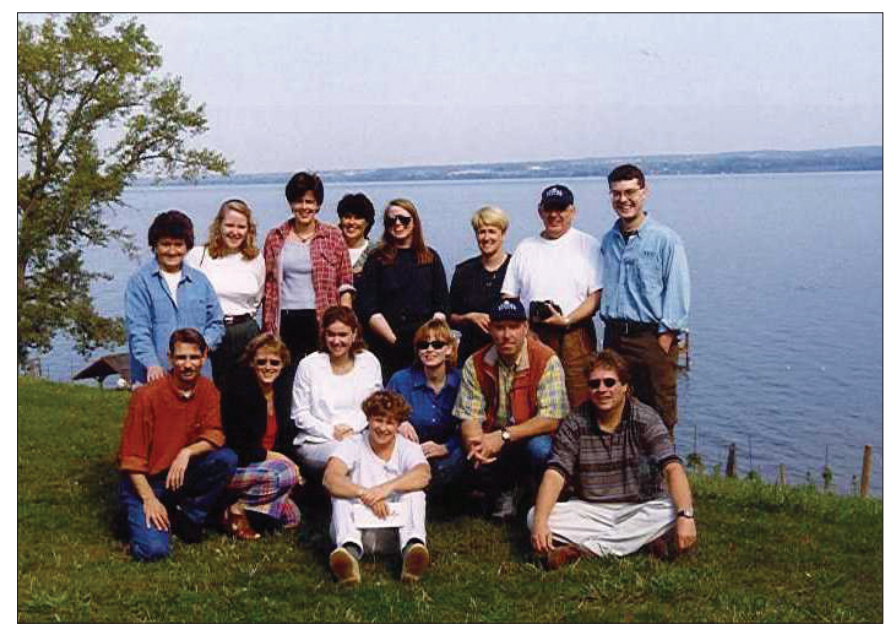

Critical care nurses from Rochester and Rotterdam 'Working Together - Achieving More' with the Finger Lakes in the background.

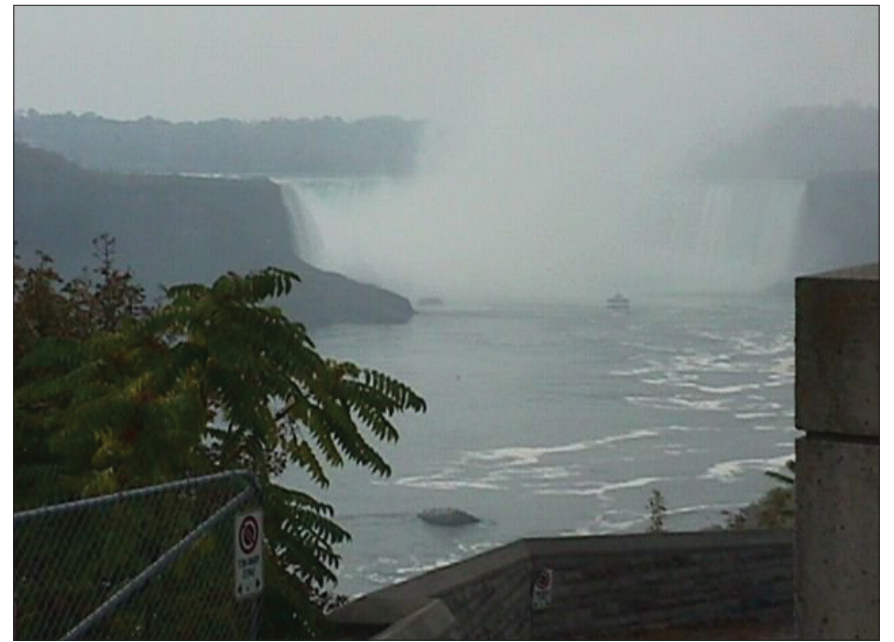

The Dutch critical care nurses visited the Niagara Falls at the end of the exchange.

evening, there is always a special farewell dinner, at which speeches and thank-you presents are given by hosts and participants to each other.

\section{Saturday}

On Saturday morning the entire group meets between 8 and 9 am at the entrance of the hospital being visited. The exchange programme is finished and everybody is leaving to go back home. Saying goodbye to someone who has hosted you for a week can be emotional, and shows that 'a nurse is a nurse wherever you are in the world'.

\section{CONCLUSION}

Looking back after four years of the exchange programme, we conclude that it is possible to start a professional exchange programme for critical care nurses in which nurses from two hospitals visit each other. It takes a commitment to start such a program. Communication therefore is a critical issue in the preparation of the week. Although the USA and the Netherlands are called western countries, a YES in The Netherlands is sometimes different compared to a YES in the USA. After each exchange, the visiting participants have said that the programme is a perfect mix in which personal, professional and social developments can be achieved. The plan for the future is to create exchange visits for longer periods, to do more research in the area of nursing practice and to extend the programme to nurses of other specialities.

Next year it is the fifth anniversary of the exchange program. One of the basic components of the program is the professional exchange between cultures. So far approximately 40 critical care nurses have participated in the program (20 from the Netherlands and 20 from the USA). It was thought after five years there should be a reunion in another country in which also critical care nurses participated of that country. Meeting halve way was difficult to decide were. As Iceland meets this criteria the most, June 2003 it is planned to celebrate our 5th anniversary there. It will be good to share our critical care practice with critical care nurses from again another country and discuss again the commonalties and the differences. Besides the professional issues there will lots of opportunities to have a look in an Icelandic critical care unit and to talk with Icelandic critical care nurses. As the meeting will take only a couple of days there will be lots of time left to see and explore some of the beautiful Icelandic nature. 


\section{Other staff in ICU}

In Iceland, there is a long tradition of help from 'nurses assistants'. These assistants help with patient care and will have received three-years of related training in college. There are also specialised staff who take care of the equipment and cleaning and washing the unit, both daily and between patients.

In the UK, we observed that many jobs done by our assistants were in fact part of an ICU nurse's job in the UK. However, in the UK but not in Iceland, there were porters, who were always available to help with transporting patients between wards. Furthermore, in the paediatic ICUs in the UK, there were special technicians who help set up all equipment, such as the ventilators and machines for continuous renal replacement therapies; the technicians also helped with transporting patients.

\section{Patient group}

In general, the patient group was more specialised in UK ICUs than in Iceland. This is because of the size of the population, so that in Iceland's ICUs, there is more variety in the patient group, both regarding age and the patient's illness and condition.

\section{Standard of nursing care}

In our opinion, in Iceland's ICUs, the standards of care were higher in many aspects of nursing care, such as patient hygiene, dressing changes and cleanliness of the nursing environment.

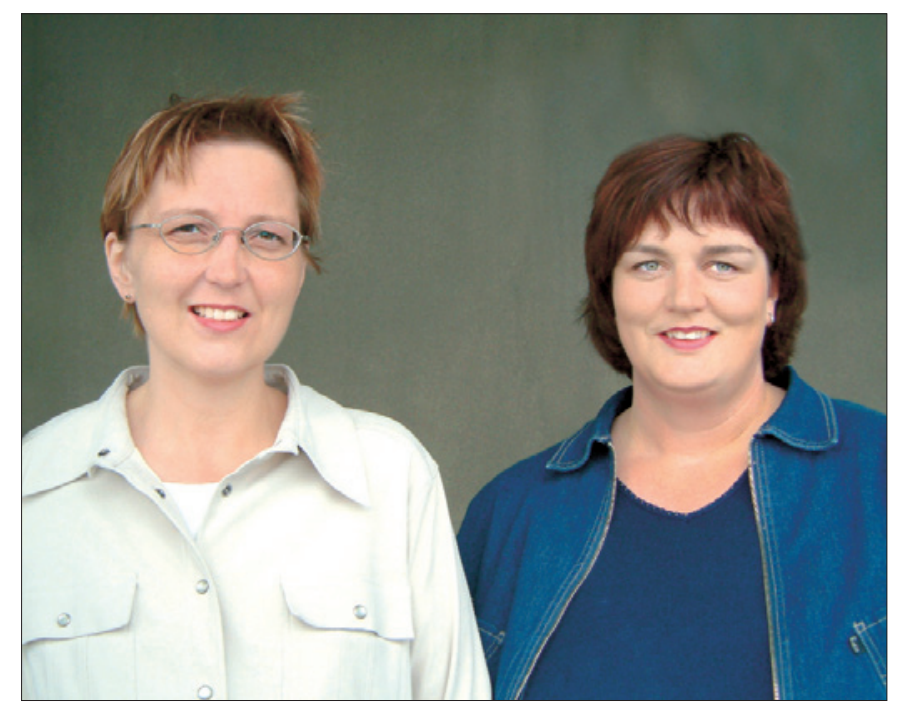

\section{Death of a patient}

When a patient dies, the care is a little bit different in Iceland. The nurses wash the patient and put him or her in special clothes. If needed by the relatives, there are priests who we can always call for support and guidance and often they have a moment with prayer beside the patient's bed. After the family and relatives have said goodbye, we put one sheet around the patient's body, with another sheet over, before transferring them to the mortuary bed. In the UK, however, we found it rather cold that the dead body should be placed in a plastic bag before being taken to the mortuary.

\section{Equipment}

The ICUs in the UK were well-equipped, with equipment very similar to ours. The most different aspect in equipment was the beds, especially those used on general wards. We found the UK beds rather primitive and not as patient- and staff-friendly as those we are used to.

\section{Drug administration}

There were differences in drug administration. In the UK, all medicines must be double-checked by another nurse before being administered. Nurses are also required to attend special courses before they can give medicines intravenously. In Iceland, nurses are allowed to give all medicines, following a course in drug administration given during training as nurse students.

\section{Medical staff}

Icelandic doctors go abroad to different places in Europe and USA to obtain further education in their speciality. They come back with different experiences and various opinions and views, all of which influence training and treatments in our hospitals and have an effect on best practice.

In the UK, doctors take decisions earlier to reduce treatment and seem to accept more easily than doctors in Iceland if a patient is not responding to the treatment given.

\section{CONCLUSION}

In general, there are no major differences in critical care nursing between Iceland and the UK. There are always some differences between countries that can be related to culture and tradition. However, the basics of ICU nursing care appear to be similar in Iceland and the UK.

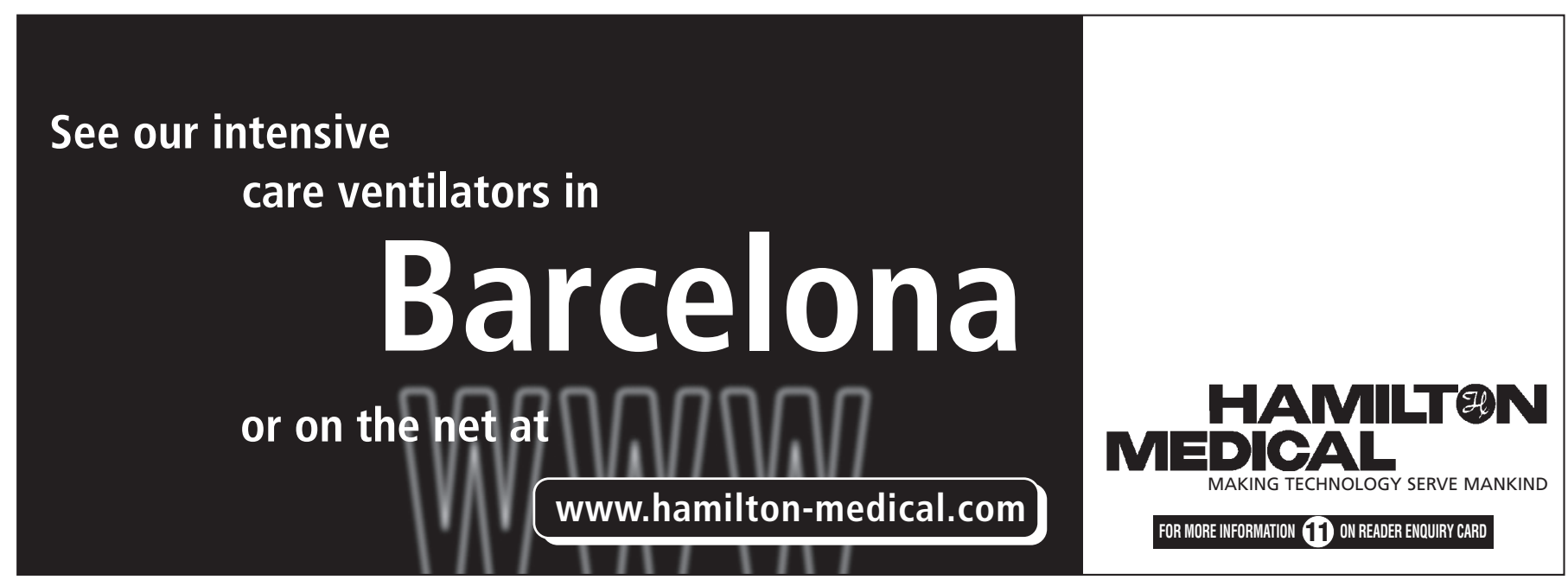

